\title{
Crossed Optical Fiber Sensor Arrays for High-Spatial-Resolution Sensing: Application to Dissolved Oxygen Concentration Measurements
}

\author{
M. Veronica Rigo and Peter Geissinger \\ Department of Chemistry and Biochemistry, University of Wisconsin-Milwaukee, 3210 North Cramer Street, \\ Milwaukee, WI 53211, USA \\ Correspondence should be addressed to Peter Geissinger, geissing@uwm.edu
}

Received 17 January 2012; Revised 11 March 2012; Accepted 14 March 2012

Academic Editor: Jiri Homola

Copyright ( $) 2012$ M. V. Rigo and P. Geissinger. This is an open access article distributed under the Creative Commons Attribution License, which permits unrestricted use, distribution, and reproduction in any medium, provided the original work is properly cited.

Optical fiber sensors using luminescent probes located along an optical fiber in the cladding of this fiber are of great interest for monitoring physical and chemical properties in their environment. The interrogation of a luminophore with a short laser pulse propagating through the fiber core allows for the measurement of the location of these luminophores. To increase the spatial resolution of such a measurements and to measure multiple analytes and properties in a confined space, a crossed optical fiber sensing platform can be employed. Here we describe the application of this platform to measuring the concentration of dissolved oxygen. The sensor is based on luminescence quenching of a ruthenium complex immobilized in a highly crosslinked film and covalently attached to the optical fibers. Both luminescence-intensity and luminescence-lifetime changes of the sensor molecules in response to changes in the concentration of oxygen dissolved in water are reported. For luminescence-intensity measurements, a second adjacent sensor region is employed as reference to account for laser pulse energy fluctuations. Enhanced quenching response in water is demonstrated by the use of organically modified poly(ethylene glycol) precursors, which increase the hydrophobicity of the film surface.

\section{Introduction}

Optical fiber sensors using luminescent probes located along an optical fiber in the fiber cladding and in close proximity of the fiber core have been of great interest for spatially resolved measurement of physical and chemical properties along a single fiber in the sensor environment [1-3]. The location of an emitting luminophore along a fiber can be determined by measuring the time delay between a short exciting laser pulse propagating in the fiber core to the sensor site and the subsequent sensor luminescence pulse returning to the front end of this fiber. The spatial resolution of these measurements is limited by the sensor luminescence lifetimes, because a minimum separation of the luminophores is required to resolve returning light pulses. However, by using a second fiber, which forms orthogonal junctions with the first fiber at all sensor sites, as an optical delay line, the minimum spacing between adjacent sensor regions can be well below the luminescence lifetime limit [4]. Because the coupling between the two fibers is evanescent, the attenuation of the excitation pulse is low, making long arrays of sensor regions feasible.

To test the performance of the crossed-fiber sensor arrays for sensing in aqueous environments, we fabricated a crossed-fiber dissolved-oxygen sensor. Dissolved oxygen (DO) is an important parameter in biological, biomedical, and industrial systems. This widespread need has led to the development of a number of water-soluble, noninvasive, sensitive, and selective detection methods that are capable of real-time DO sensing. In the past, oxygen sensors have mainly been based on amperometric Clark-Type electrodes [5]. Within the past few years, research in optical sensors have overcome most of the disadvantages of amperometric 
sensors; in contrast to the latter, optical sensors are free of electromagnetic interference, do not consume oxygen and, in general, respond faster [6-9].

Different optical sensors have been reported [10-16] for oxygen measurement in both gas and aqueous environments. Most of these sensors respond to changes in the luminescence intensity of an indicator dye; however, as with all measurements based on luminescence-intensity changes, these measurements are hampered by the fact that signal changes may arise from a number of factors other than the measurand. This problem could be overcome either by using luminescence-lifetime detection (using either direct timeresolved detection or the phase-shift method) [12, 17-19] or by employing ratiometric sensors $[20,21]$. In the latter case the ratio of the indicator-luminescence intensity and a reference-luminescence intensity can compensate for system fluctuations; in spite of the need for a reference, measurements based on indicator-luminescence changes are considered preferable to measurements based on luminescencelifetime changes, because the latter require a more sophisticated experimental setup.

The use of luminescent ratiometric sensors combined with the optical fiber technology provides an opportunity of real-time and remote DO measurement. Fiber-optic oxygen sensors are frequently of the extrinsic type [22-28], with a single sensor mounted at the distal end of the fiber. This allows for point monitoring; however, for measurements at multiple locations, separate fibers are needed for each additional sensor. Thus, placing luminescent sensors placed into the fiber cladding (or a suitable material replacing the original fiber cladding) along a continuous stretch of fiber (distributed sensing) or in many discrete regions (quasidistributed sensing) constitutes an efficient alternative; when appropriate fiber-guiding conditions are maintained at the sensor regions [4], the interaction of the probe light propagating in the fiber core and the luminosensor in the cladding is solely through the evanescent fields of the core modes.

The DO sensor implemented here consists of the sensor dye tris(1,10-phenanthroline)ruthenium(II) chloride, $\mathrm{Ru}\left[(\mathrm{Phen})_{3}\right] \mathrm{Cl}_{2}$, immobilized in a poly(ethylene glycol) (PEG) matrix. This polymer replaces the optical cladding, which is removed prior to covalent attachment of the PEGfilm. The immobilization of this ruthenium complex within an optically transparent, low-refractive index and oxygenpermeable structural matrix is of primary concern for luminescent oxygen fiber-based sensors. Issues regarding oxygen permeability and luminophore solubility within the matrix, luminophore leaching, and complex-dependent luminescent quenching were considered. We measured sensor calibration curves and characterized the sensor with respect to response time, detection limit, repeatability, biocompatibility, longevity, and possible interferences.

\section{Background}

Our optical sensor consists of an optical fiber with exposed core, PEG-films containing the sensor and reference molecules, and a sensing medium. The versatility of the PEGpolymer synthesis enables optimization of sensor parameters (e.g., sensitivity, response time, and signal-to-noise ratio) via control of film properties such as coating length, thickness, and porosity. The PEG films replace the original cladding material only at the sensor/reference regions. The refractive index of the cladding is such that the guiding condition of the fiber is maintained, that is, total internal reflection of the light propagating in the fiber core is taking place at the core/cladding interface at the sensor regions. The interaction of light propagating in the fiber core, which is used to interrogate the sensor, and the indicator molecules in the cladding are through evanescent fields, which decay exponentially with distance from the core/cladding interface. This defines a short-range sensing volume within which the evanescent field may interact with the indicator molecules [29]. Sensor molecules within the range of evanescent fields may absorb light of the appropriate frequency, attenuating the total internal reflection [29]. Also, the luminescence of an indicator molecule may be coupled back into a guiding mode of the fiber via the evanescent fields.

The spatial information regarding the location of an emitting luminophore along the fiber can be obtained using optical time-domain reflectometry (OTDR) [30-33] or, more appropriately for luminescence transduction, optical time-of-flight detection (OTOF) [34]. Laser pulses fed into the front end of the fiber cause pulsed evanescent excitation of the luminophores [35]. The luminescence pulses returning to the front of the fiber arrive with a time delay $\Delta t_{d}$ that allows for the calculation of the location $L$ of the emitting luminophore. In our setup, the luminescence is captured by a second fiber (referred to as "detection fibers") at right angle to the "excitation fiber" at every sensor region. The second fiber provides the time delay necessary to temporally resolve the signals originating at the sensor regions as seen in Figure 1 (for details see $[36,37]$ ). This allows for an increased spatial resolution in multisensor arrays, as we demonstrated with an array of 100 crossed fiber junctions in a space of $6 \mathrm{~cm} \times 6 \mathrm{~cm} \mathrm{[38].} \mathrm{This} \mathrm{work} \mathrm{is} \mathrm{part} \mathrm{of} \mathrm{our} \mathrm{effort} \mathrm{to}$ develop sensing elements (i.e., individual sensor junctions) for this sensor-array architecture; the long-term goal is to incorporate various sensing elements for different sensing tasks into this architecture.

Optical oxygen sensors are commonly based on the ability of molecular oxygen to quench the luminescence of certain transition metal complexes [39] or porphyrin derivatives [40]. The variation of the luminescence signal, $I$ (and the luminescence decay time $\tau$ ) with the dissolved oxygen concentration DO, is described by the Stern-Volmer equations [41]

$$
\frac{I_{0}}{I}=\frac{\tau_{0}}{\tau}=1+K_{\mathrm{SV}} \mathrm{DO}
$$

where $I_{0}$ and $\tau_{0}$ are the respective luminescence intensity and luminescence lifetime in the absence of oxygen. The Stern-Volmer quenching constant $K_{\mathrm{SV}}=k_{q} \tau_{0}$ depends on the diffusion-dependent bimolecular quenching constant $k_{q}$, which in turn is given by $k_{q}=4 \pi a D\left(10^{-3} N_{A}\right)$. $D$ is the sum of the diffusion coefficients of oxygen and luminophores, $a$ is the sum of the molecular radii, and $10^{-3} N_{A}$ is the number 


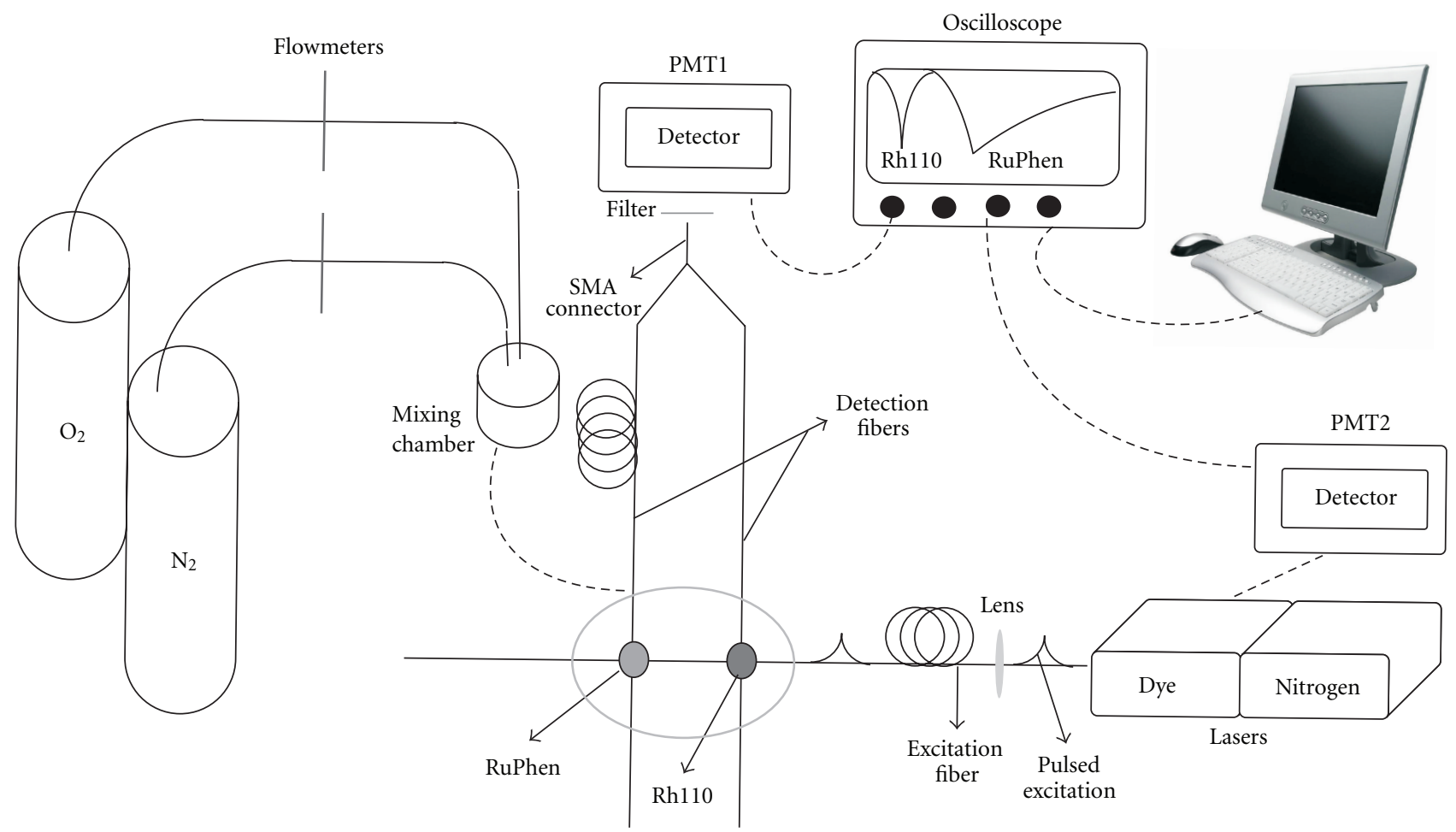

FIGURE 1: Experimental setup showing the crossed-fiber sensor system. The grey ellipse surrounding the fiber-fiber sensor junctions represents the flow cell in which the sensors are enclosed.

of molecules per millimole [42], where $N_{A}$ is Avogadro's number.

The ruthenium complex, $\mathrm{Ru}\left[(\mathrm{Phen})_{3}\right] \mathrm{Cl}_{2}$, was chosen for this work as it exhibits high oxygen sensitivity because of its long unquenched lifetime $\tau_{0}(\sim 0.8 \mu$ s in ethanolic solution). Furthermore, it absorbs strongly at blue-green wavelengths and has a relatively large Stokes shift. As seen from (1), oxygen pressure may be measured by monitoring luminescence intensity or luminescence lifetime. We have adopted both approaches to DO determination.

\section{Experimental}

3.1. Reagent and Materials. The complex tris(1,10-phenanthroline)ruthenium(II) chloride hydrate (hereafter referred to as "RuPhen"), poly(ethylene glycol) diacrylate (PEG-DA) with an average molecular weight of 575 and, poly(ethylene glycol) dimethacrylate (PEG-MA) with a molecular weight of 1000, 2,2-dimethoxy-2-phenylacetophenone (DMPA), 3(trichlorosilyl) propyl methacrylate (TPM), trimethylolpropane triacrylate (TPT), sulfuric acid, and hydrogen peroxide (30\% V/V in water) were obtained from Sigma Aldrich Chemical Company (Milwaukee, WI). The laser dye Rhodamine 110 (Rh110) was purchased from Exciton Corporation (Dayton, $\mathrm{OH}$ ). Deionized water (DI) with a resistivity of $18 \mathrm{M} \Omega \cdot \mathrm{cm}$ was used for all aqueous experiments. All reagents were used as received without further purification.

3.2. Chemical Modification of the Fiber Core. All setups used multimode silica fibers with a TECS (trademark of
$3 \mathrm{M}$ Corp.) cladding (FT-200-UMT with core diameter of $200 \mu \mathrm{m}$ ) and SMA 905 connectors purchased from ThorLabs, Inc. (Newton, NJ). The fibers had core and cladding refractive indices of $n_{\mathrm{co}}=1.457$ and $n_{\mathrm{cl}}=1.404$, respectively. The sensor regions themselves were prepared by first removing the fiber jackets of both fibers comprising the fiber junction by mechanical stripping. Subsequently, the cladding layers were removed by rubbing with a cotton swab immersed in acetone and the exposed core was cleaned using Piranha solution ( $3: 1$ sulfuric acid: hydrogen peroxide) to eliminate any organic residue. The first step in the synthesis of the new cladding was treating the silica core of the fiber with $1 \mathrm{M}$ nitric acid solution to activate the hydroxyl groups of the silica. Then, a silanation reaction was carried out by exposing the fiber core surface with a $1 \mathrm{mM}$ solution of TPM in a nitrogen atmosphere, at $18^{\circ} \mathrm{C}$ for $1 \mathrm{hr}$ [43-45]. Once the reaction was completed, the fiber was removed from the reaction cell, washed with DI water and ethanol, and dried in a nitrogen stream. The chlorosilane modification of the core is necessary to create gels that adhere covalently to the fiber surface.

3.3. Sensor Preparation. For molecules physically entrapped in polymers, thin PEG-based films were synthesized by mixing $1 \mathrm{~mL}$ of PEG-DA 575 with $10 \mathrm{mg}$ of DMPA to prepare the PEG-DA 575 precursor solution, which was then diluted with water to make a $40 \% \mathrm{~V} / \mathrm{V}$ solution (referred as coating solution). Separate solutions of $7.0 \mathrm{nM}$ RuPhen complex (i.e., the oxygen sensor molecule) and $1.3 \mathrm{nM}$ Rh110 (the intensity reference molecule) were made in ethanol. Each luminophore solution was added separately to the coating 
solution to make a $1: 10(\mathrm{~V} / \mathrm{V})$ mixture (designated as doped-coating solution). Highly crosslinked PEG-DA (TPTPEG-DA 575) precursor solution was synthesized by adding, while stirring, $10 \mathrm{mg}$ of DMPA and $300 \mu \mathrm{L}$ of TPT to $1 \mathrm{~mL}$ of PEG-DA 575 and further sonicated for $10 \mathrm{~min}$. The coating solution was formulated by diluting this mixture with water to make a $40 \% \mathrm{~V} / \mathrm{V}$ solution. Subsequently the luminophore solution (either RuPhen or Rh110) was mixed with the $40 \%$ (V/V) coating solution to prepare a final 1:10 (V/V) dopedcoating mixture. Additional PEG films were prepared using PEG-MA with a molecular weight of $1000 \mathrm{~g} / \mathrm{mol}$. Iin this case, first the waxy solid was heated at $37^{\circ} \mathrm{C}$ to melt the polymer and then $1 \mathrm{~mL}$ of the liquefied reagent was used to prepare the precursor solution.

After preparing the doped-coating mixtures, the fibers were placed in the flow cell such that orthogonal fiber-fiber junctions were created (see Figure 1) with the TPM-modified fiber cores just touching. The first sensor region in the array (i.e., the region that is excited first by the laser pulse) contains the intensity reference molecule Rh110. The RuPhen oxygen sensor is located in the second fiber-fiber sensor junction. $2.0 \mu \mathrm{L}$ of each doped-coating mixture were placed in the corresponding region using an automatic pipette and the gel formation was initiated upon UV-exposure $(365 \mathrm{~nm})$ of the doped-coating mixture [46-50]. The free-radical copolymerization of methacrylate and terminal acrylate groups between the PEG derivatives (of the doped-coating mixture) and the TPM functionality (bound to the fibercore) creates polymer films covalently attached to the fiber core and where the luminophore is physically entrapped in the gel matrix. A Photon Technology International (PTI) 150-Watt Xenon lamp (Birmingham, NJ) with a narrow bandpass interference filter (J43-057-Edmund Optics) was used to cure PEG-gel films in approximately 1 minute. After gel formation the flow cell was filled with deionized water.

3.4. Instrumentation. A typical setup used in these experiments is shown in Figure 1. A dye laser PTI PL201 (using the laser dye Coumarin 460-Exciton Corporation) pumped by a nitrogen laser PTI PL2300 (pulse width $0.6 \mathrm{~ns}$, pulse energy $1.4 \mathrm{~mJ}$, pulse repetition rates of $3-10 \mathrm{~Hz}$ ) served as the excitation source for the sensor array system. A pair of lenses was employed to couple the light into the excitation fiber. The sensors were mounted in a home-built flow cell filled with deionized water. Oxygen and nitrogen mixtures from pure gas cylinders (Praxair grades 2.6 and 4.8, resp.) were used to achieve the range of DO concentrations by bubbling these gas mixtures into the flow cell. The gases were premixed in a separate mixing chamber and delivered through $1 / 4$-in.-i.d. flexible PVC tubing into the flow cell.

The luminescence emitted by the sensor molecules was captured by the fibers crossing at right angle to the fiber carrying the excitation light pulse and guided to the detector. A narrow bandpass filter (610 nm Edmunds J43-079) was used in front of the Burle C31034A photomultiplier tube (PMT1; with Peltier cooling) to select the RuPhen luminescence and the Rh110 reference pulses. As seen in Figure 1, the array is designed such that the region containing the reference is excited first; also, the lengths of the detection fibers are chosen such that the emission of the Rh110 reference arrives first at PMT1. The RuPhen luminescence pulse is delayed by passage through an additional stretch of fiber $(9 \mathrm{~m})$ to arrive at PMT1 after the reference signal has subsided. Thus, the output current of PMT1, which was analyzed with a LeCroy LC564DL digital storage oscilloscope (DSO) with a bandwidth of $1 \mathrm{GHz}$ and sampling rate of $4 \mathrm{GS} / \mathrm{s}$, displays both region signals sequentially in one trace. A trigger for the DSO was generated using a second PMT2 (RCA 1P28, 1.6ns rise time), which collected light scattered from the front of the excitation fiber. A LabVIEW (National Instruments) program was written to collect and analyze the data. All the experiments presented here were repeated from three to four times, and the peak ratios were measured and averaged. The RuPhen referenced intensity $\widetilde{I}\left(I_{\text {RuPhen }} / I_{\text {Rh110 }}\right)$ is calculated as the luminescence integrated intensity of RuPhen itself divided by the luminescence integrated intensity of the reference dye.

\section{Results and Discussion}

4.1. Polymer Selection and Indicator Leaching Test. For oxygen sensing in aqueous media, the polymer matrix must have high oxygen permeability to allow the rapid interaction of the dissolved oxygen with the indicator entrapped in that matrix, must be of mechanical and chemical stability, and must be optically clear. It is also worth noting that the low oxygen solubility in water at ambient conditions and the lower DO diffusion coefficient compared to the one in gas phase result in a poor sensitivity and response to DO. For PEG-based gels it has been demonstrated that their mesh size can be increased by the introduction of water to the precursor solution, which reduces the degree of polymer cross-linking [51]. An increase in porosity allows for faster diffusion of analytes through the gel; however, indicator leaching is increased. Leaching is an important aspect in the preparation of water-compatible sensors. The performance, repeatability, and the response of such sensors are associated to the degree of leaching. Several factors will determine the extension of the leak; with respect to the indicator molecules their molecular size, their solubility in both water and polymer matrices have to be considered for the fabrication of aqueous-phase sensors. With regard to the matrix polymer its chosen porosity is a compromise between indicator-molecule leaching from the solid matrix to the aqueous medium and analyte diffusion from aqueous environment to the entrapped molecule. Another important restriction in the selection of a polymer matrix is its level of hydrophobicity. Based on the molecular size of Rh110 and RuPhen the best matrix candidates are PEG-DA with a molecular weight of 575 or/and PEG-MA with a molecular weight of 1000 . The higher the polymer molecular weight is, the more hydrophobic and porous it becomes.

Using these polymers in the design of aqueous sensors, we tested the degree of indicator leaching associated with them. For this, the change in luminescent intensities of the two regions placed in a water-filled flow cell was monitored every 10 minutes for 2 days. Figure 2 curves (a) and (b) 


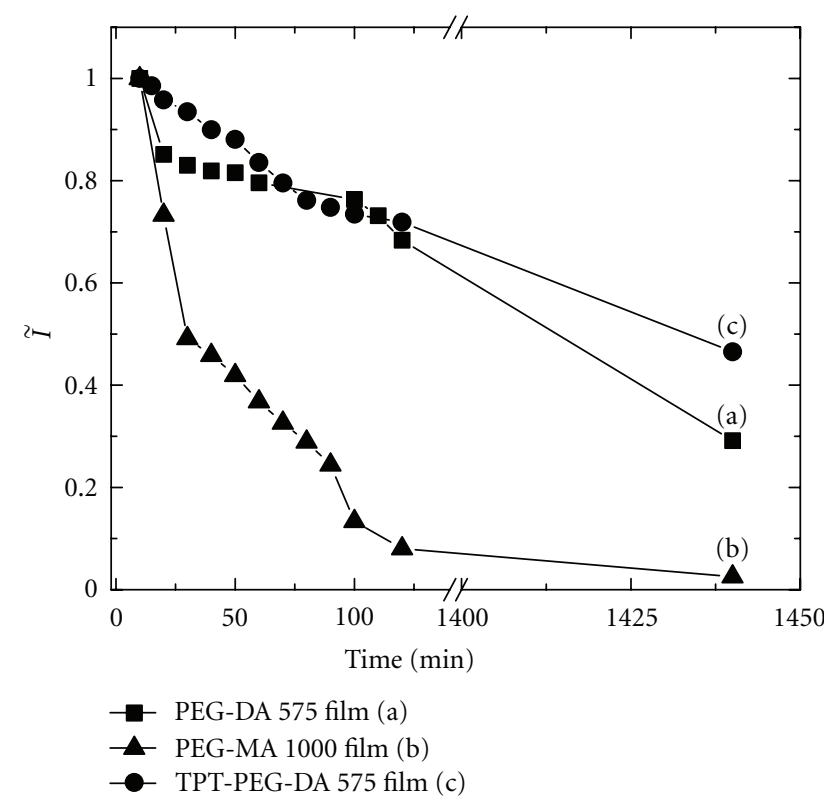

Figure 2: Luminophore leaching for different cladding materials: referenced-RuPhen intensity decrease versus time, which occurs because of RuPhen leaching from films made with the following polymers: (a) PEG-DA 575 (b) PEG-MA 1000, and (c) PEG-DA 575 tailored with TPT. The leaching percentages are (a) $71 \%$, (b) $89 \%$, and (c) 50\%. The initial values of each curve were normalized to one for better comparison.

show the integrated referenced RuPhen intensity as function of time for sensors based on PEG-DA 575 and PEG-MA 1000 polymer films, respectively. The observation of significantly larger leaching from the PEG-MA 1000 film suggests an increase in indicator-molecule leaching as the matrix molecular weight increases. This can be understood in term of two factors: first, it appears that PEG-MA 1000 is not sufficiently hydrophobic to retain RuPhen in its matrix and to avoid its diffusion to the aqueous medium. The second factor is the larger porosity obtained with PEG-MA 1000: the larger the mesh size the faster is the indicator-molecule diffusion toward the aqueous medium. Even though leaching is considerably less for a PEG-DA 575 matrix, the loss of 71\% of RuPhen is quite detrimental to sensor performance. An alternative for decreasing indicator leaching is the incorporation of alkyl groups in the polymer structure $[13,52-54]$ : this provides not only high porosity (because of the bulky groups) but also hydrophobicity. Therefore, we modified the PEG-DA 575 polymer by adding a hydrophobic crosslinker, TPT. The incorporation of this trifunctional monomer to the PEG-DA 575 precursor mixture improves the resistance to indicator leaching by more than $20 \%$, as shown in Figure 2 curve (c). Even though an intensity loss of $50 \%$ is quite large, we still can detect sufficient signal intensity from the retained sensor molecules. Increasing the TPT concentration further results in an increase in the coating-solution refractive index, which will lead to refractive losses at the junctions and eventually to the loss of guiding condition of the fiber. After the initial leaching ceased, the TPT-PEG-DA 575-based DO

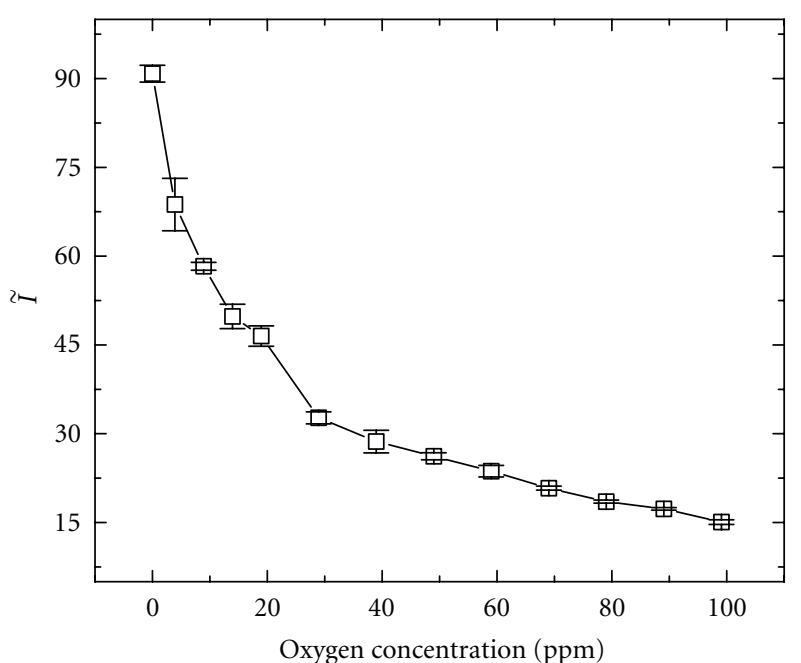

Figure 3: RuPhen intensity referenced to the Rh110 intensity as a function of DO concentration in ppm.

sensor shows excellent stability, which is described in the following sections. While for this sensor one goal was to find a PEG formulation that would provide physical entrapment of the dye which minimized leaching but not overly compromised sensor response time, covalent attachment of the sensor dye to the PEG matrix is an alternative approach to control leaching, which we employed in other work [55].

4.2. Quenching Sensitivity. The sensor sensitivity can be expressed by the overall quenching efficiency $\left(E_{\mathrm{DO}}\right)$ given by

$$
E_{\mathrm{DO}}=\frac{\tilde{I}_{\mathrm{N} 2}-\tilde{I}_{\mathrm{O} 2}}{\tilde{I}_{\mathrm{N} 2}} \times 100 \%
$$

where $\widetilde{I}_{\mathrm{N} 2}$ and $\widetilde{I}_{\mathrm{O} 2}$ represent the referenced RuPhen intensities in fully deoxygenated and fully oxygenated water, respectively.

The oxygen concentrations in air-saturated and oxygensaturated solutions were calculated to be 8.5 and $99.1 \mathrm{ppm}$ at $22^{\circ} \mathrm{C}$, respectively. This calculation was based on the solubility equation of oxygen in water [56]. For each DO concentration, 1000 luminescence pulses were averaged. All measurements were performed at room temperature. Figure 3 shows the response of the TPT-PEG-DA 575-based oxygen sensor to different DO concentrations.

Applying (2) to the data shown in Figure 3, we obtained a value of $E_{\mathrm{DO}}=84 \%$. This shows that, because of the issues discussed in the Introduction, the quenching response of the oxygen sensor is reduced in the aqueous phase compared to the gas-phase value of $E_{\mathrm{DO}}=98 \%$ (found in our laboratory [57]). However, the measured quenching efficiency still constitutes a sufficiently high overall quenching response to attain good sensor resolution, especially at low oxygen concentrations. The DO-sensor described by $\mathrm{Xu}$ et al. [9] used $\mathrm{Ru}(\mathrm{II})$-tris(4,7-diphenyl-1,10-phenanthroline) as sensor molecule enclosed in a sol-gel matrix and ratiometric detection exhibited a DO quenching efficiency of $\sim 92 \%$. 


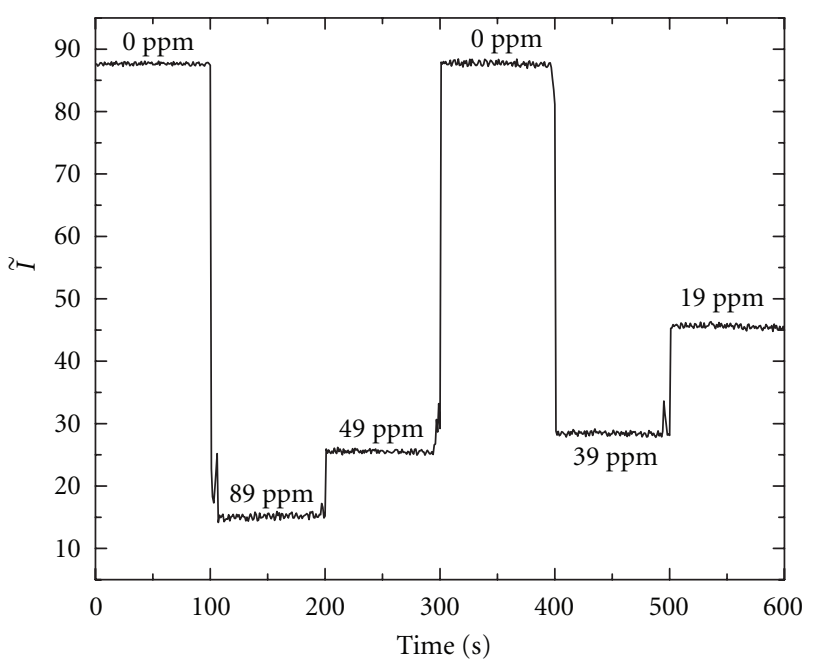

FIGURE 4: Reversibility of the sensor response to DO changes.

The sensor studied by Koo et al. [13], which employed platinum (II) porphyrins derivatives incorporated in organically modified silicate nanoparticles, attained a value of $E_{\mathrm{DO}}=$ $97 \%$. Our sensing system has a somewhat lower quenching performance, but the sensors mentioned above are for use in confined areas such cells, where our sensor, which has the potential for remote monitoring, is not applicable.

4.3. Sensor Reversibility. We used the cycle-purging method to check the reversibility of TPT-PEG-DA 575 films with RuPhen physically entrapped in this highly crosslinked matrix. The referenced sensor intensity $(\widetilde{I})$ is plotted in Figure 4 for varying oxygen concentrations. Upon switching from nitrogen-saturated to oxygen-saturated solution, $\widetilde{I}$ decreased with increasing DO concentration. This process was repeated multiples times with no significant change in $\widetilde{I}$ between the initial nitrogen-saturated solution, indicating complete reversibility of the sensor going from high to low DO concentration and vice versa. In addition, this sensor showed at least a $98 \%$ recovery each time after changing the environment, implying a good reproducibility.

Over the range of oxygen concentrations that we studied and for the measurement duration, we did not observe significant photobleaching of the Ru complex, which in turn may indicate singlet oxygen created in the quenching process can rapidly diffuse away before causing oxidative damage, as was reported in [58].

4.4. Sensor Response Time. The sensor response time was tested by alternating oxygenation/deoxygenation cycles, which is shown in Figure 5. The calculated transition time is of the order of 5-7 seconds; however we believe that the sensor itself has a shorter response time because the measured response time includes the time required to change between oxygenated and deoxygenated environments in the flow cell and the attached tubing. Therefore the reported value of the response time is an upper limit of the actual one. The thickness of the PEG film plays an important role in the

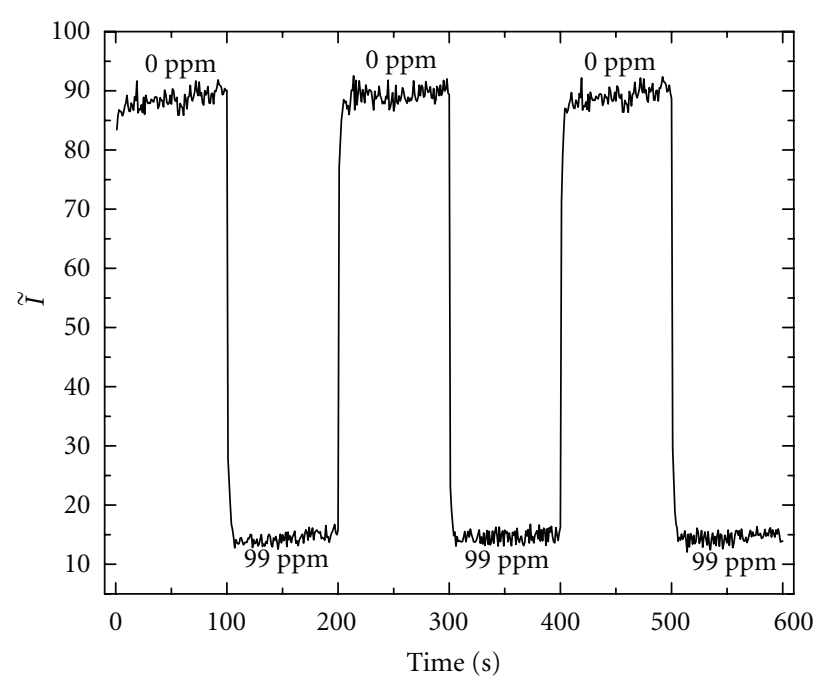

FIGURE 5: Sensor response to switching from nitrogen-saturated to fully oxygenated environments.

sensor response time, particularly in the highly crosslinked films used here, which could reduce analyte diffusion to the sensor molecules and negatively impact the sensor response time. The crosslinking density in the PEG films used here still allows for effective analyte diffusion into the sensing volume between the fibers; the total film thickness through which the analyte must travel to reach the sensing volume is estimated to be less than $1 \mathrm{~mm}$. Should faster response times be required, the creation of highly porous files using a microtemplating approach in combination with covalent attachment of the sensor dye would offer an alternative $[55,59]$.

4.5. Sensor Calibration and Linearity. The RuPhen luminescence quenching by oxygen is described by the Stern-Volmer equations (1), which are used to estimate the linear range of our sensor. Figure 6 shows Stern-Volmer plots of the RuPhen referenced intensity and luminescence lifetime versus DO concentration for the TPT-PEG-DA 575-based sensor; the dotted line represents the best linear fit generated by the least-squares method. The sensor has a good linearity over the whole range of DO concentrations tested, which may indicate negligible matrix heterogeneity effects.

The good $E_{\mathrm{DO}}$ and linearity in the Stern-Volmer plot may result from the structure and hydrophobic nature of the film used in the sensor preparation. The introduction of TPT may provide good hydrophobicity and sufficient porosity to create multiple channels, allowing oxygen to travel efficiently towards the sensor molecule entrapped in the matrix. Because oxygen has higher affinity for hydrophobic media, this could lead to an improvement in sensitivity as well as in linearity.

The linear fit of Figure 6 yields a Stern-Volmer quenching constant of $K_{\mathrm{SV}}=0.048 \mathrm{ppm}^{-1}$. Using the unquenched lifetime $\tau_{0}=850 \mathrm{~ns}$ (measured in the highly crosslinked film submerged in water) we obtain for the bimolecular quenching constant the value of $k_{q}=1.8 \times 10^{9} \mathrm{~L} \mathrm{~mol}^{-1} \mathrm{~s}^{-1}$, 


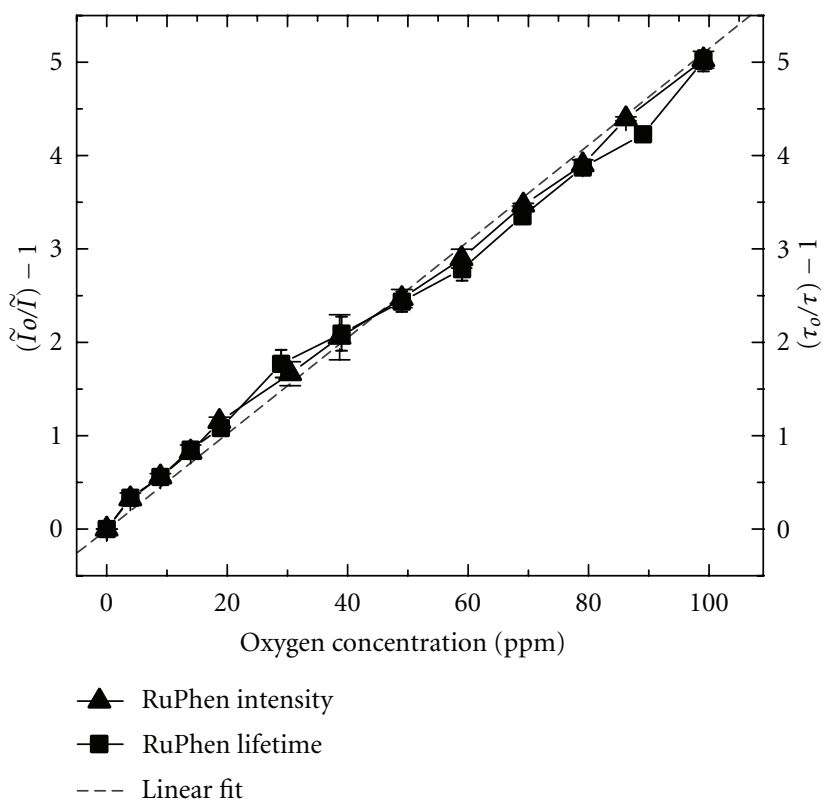

Figure 6: Stern-Volmer plots of referenced RuPhen intensity and lifetime.

which is in the same order of magnitude to the value reported for oxygen sensing with RuPhen in solution $\left(k_{q}=4.2 \times 10^{9} \mathrm{~L}\right.$ $\left.\mathrm{mol}^{-1} \mathrm{~s}^{-1}[60]\right)$.

To calculate the pressure resolution afforded by the referenced sensor, we use the sensor sensitivity defined as

$$
\Delta \tilde{I}(\mathrm{DO})=\left(\frac{d \tilde{I}}{d(\mathrm{DO})}\right) \Delta \mathrm{DO}
$$

where $\Delta \tilde{I}(\mathrm{DO})$ is measurement uncertainty of the referenced-integrated intensities at a given dissolved oxygen concentration, and $\triangle \mathrm{DO}$ is the corresponding resolution of the DO concentration. Using for the referenced-intensity uncertainty the $95 \%$ confidence band $t \times \sigma_{N}(\tilde{I})$, that is, the standard deviation of the mean $\sigma_{N}(\tilde{I})$ at a given DO concentration with the appropriate $t$-value for $n=3$, we obtain for oxygen concentrations of $3.91 \mathrm{ppm}$ and $48.98 \mathrm{ppm}$, a sensor resolutions of $0.78 \mathrm{ppm}$ and $0.82 \mathrm{ppm}$, respectively. To calculate the DO detection limit, $\mathrm{DO}(\min , \tilde{I})$ afforded by the sensor, we use the sensitivity at DO $=0$, which using (1) is given by $d \tilde{I} /\left.d(\mathrm{DO})\right|_{\mathrm{DO}=0}=-K_{\mathrm{SV}} \tau_{0}$ With $\sigma_{N}\left(\tilde{I}_{0}\right)=$ 90.83 ppm, we obtain for the detection limit $\mathrm{DO}(\mathrm{min}, \widetilde{I})=$ $179 \mathrm{ppb}$. The same calculation for the lifetime data yields for oxygen concentrations of $3.91 \mathrm{ppm}$ and $48.98 \mathrm{ppm}$ a sensor resolution of $0.41 \mathrm{ppm}$ and $0.32 \mathrm{ppm}$, respectively, and a detection limit of $140 \mathrm{ppb}$. Thus, in spite of the two evanescent coupling processes occurring in our sensor platform, the detection limit for DO is within one order of magnitude of the detection limit reported for commercially available extrinsic fiber sensors, where the sensor dyes are mounted at the distal end of a fiber and interrogated with the phase-shift method [10]. McDonagh and coworkers [54] reported a sol-gel-based optical DO sensor with a resolution of $0.18 \mathrm{ppm}$ and a detection limit of $5 \mathrm{ppb}$ suggesting that sol-gel films are better candidates for DO sensing than the modified-PEG-DA gels. We investigated the use of sol-gel matrices in our fiber sensors, but while the sensor-molecule encapsulation was successful, the sol-gel (TEOS-based gels) was not covalently attached to the fiber core, which caused a fluctuating sensor response because of (slight) respective movements of the fibers constituting a fiber-fiber sensor junction.

4.6. Interferences. As explained above, the oxygen sensing mechanism is based on the quenching of the RuPhen luminescence, which, however, may also be quenched by other species. The interference because of anions, cations, and some oxidizing agents was tested by first purging the sensor chamber with nitrogen to create at oxygenfree atmosphere. Subsequently each interfering solution was injected into the flow cell while continually monitoring the referenced RuPhen intensity in an oxygen free aqueous medium. The overall sensor quenching responses to the dissolved species are depicted in Figure 7. It can be seen that the sensor response is affected by the presence of $\mathrm{F}^{-}$and $\mathrm{Fe}^{+3}$. However, the sensor response to these interferences is much smaller than the sensor response to oxygen. The matrix thus protects the entrapped indicator from the environment, preventing interference with the luminescent properties of the sensor molecules. Without this shielding of the indicator, its luminescence would behave unpredictably in a given media, making calibration even of ratiometric luminophores difficult or impossible.

In addition to the interference studies, a DO sensor is most likely to be applied in biological media (e.g., blood, rivers, food processing, etc.) where the contamination of the sensor surface with proteins and cells could affect the diffusion of oxygen into the sensor. As a consequence, the antibiofouling properties of the TPT-PEG-DA 575 films were examined. The effects of nonspecific protein binding to the PEG-DA-film surface were investigated by the addition of bovine serum albumin (BSA, $0.3-5 \%$ (W/V) aqueous solution) to the flow-cell containing the fiber-fiber junctions immersed in DI water (see Figure 7 right-lower corner). First, the flow cell was purged with nitrogen, then, a $0.3 \%$ (W/V) BSA solution was added and allowed to interact with the fiber regions in the aqueous medium for 20 minutes. After this time period, oxygen was introduced to the flow cell with a DO concentration of $1.5 \mathrm{ppm}$. As expected the RuPhen intensity was quenched; after $\sim 75$ minutes the sensor was flushed with nitrogen and the RuPhen intensity recovered to the initials values, indicating that no protein binding on the sensor surface occurred. We observed the same trends with a BSA concentration of $5 \%$ $(\mathrm{W} / \mathrm{V})$ with the sensor maintaining its recovery time and sensitivity. The results from the nonspecific protein-binding assay show the importance of encapsulating molecules inside the protective PEG-DA matrix. The antibiofouling property, biocompatibility, and nontoxicity of poly(ethylene glycol) have been reported by different authors [61-65], making this material suitable for biological applications. The vulnerability of the sensor to metal ions, notorious oxidizing species, 


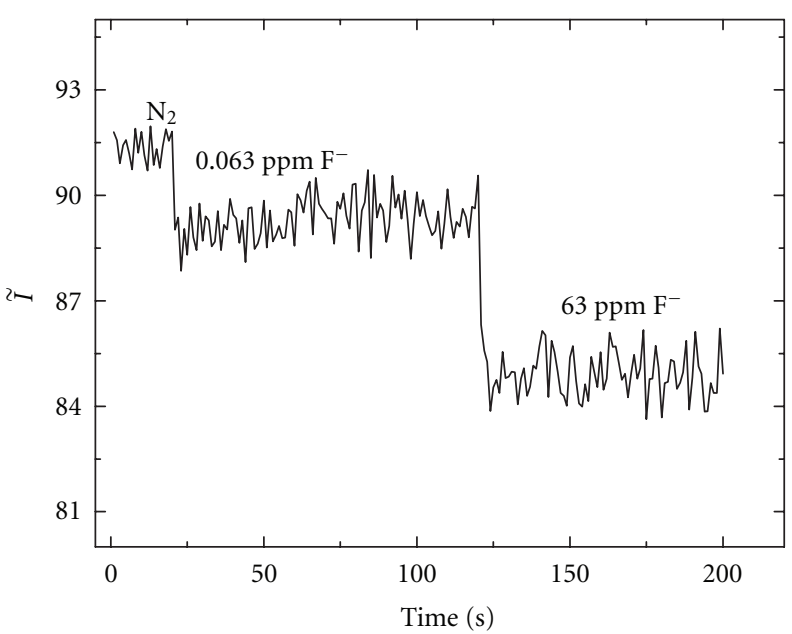

(a)

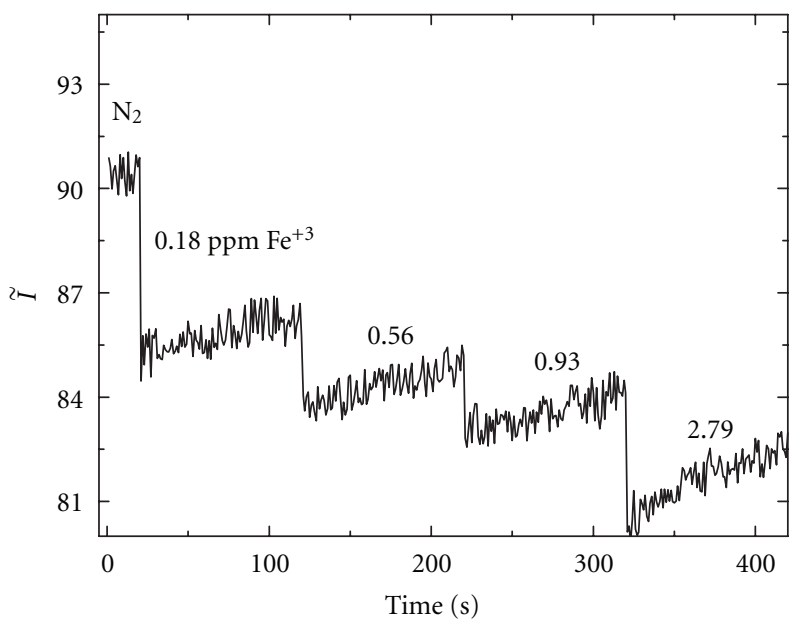

(c)

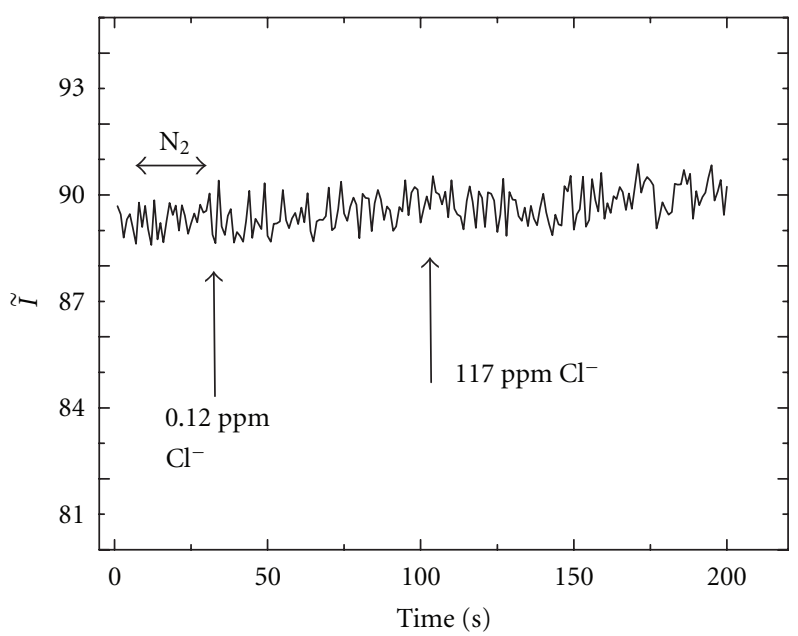

(b)

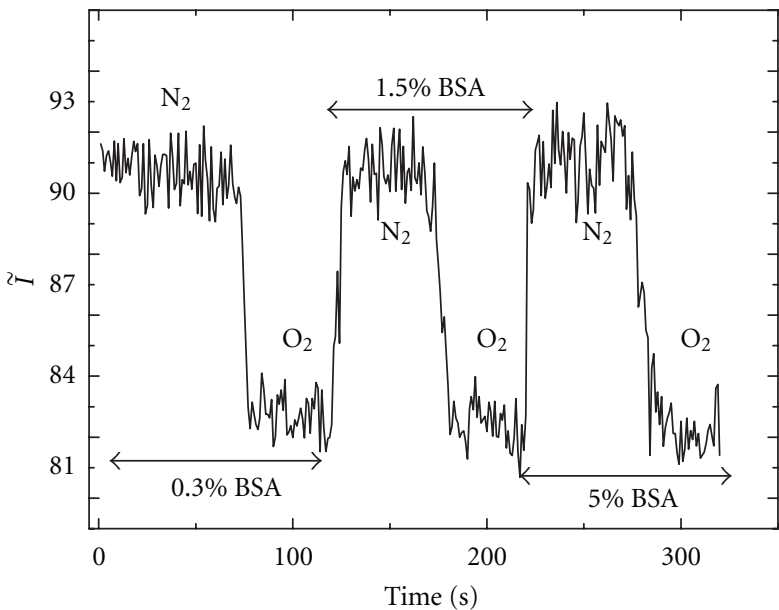

(d)

FIGURE 7: Potential interferents of the DO measurement: sensor response to the presence of ionic species and proteins.

and biological materials was prevented, demonstrating the capability of this sensor for environmental DO monitoring. The sensor performance towards DO response was also tested over time and the long-term quenching stability of DO sensor was established over a period of 6 months when the fiber-sensor array was continuously immersed in water.

\section{Conclusions}

Dissolved-oxygen sensing was implemented in a crossedfiber sensor configuration, with sensing based on luminescence quenching of ruthenium complexes entrapped in a PEG-DA matrix. In spite of the weak evanescent coupling of the two fibers in a sensor junction, both lifetimeand intensity-based oxygen measurements exhibited good repeatability and signal-to-noise ratio with a limit of detection of $\sim 0.8 \mathrm{ppm}$. With the use of second crossed-fiber junction to measure the excitation pulse intensity and referencing the ruthenium sensor signal, the intensity-based and lifetime-based measurements were of similar quality with regard to resolution and detection limit. Chemical modification of the PEG-DA matrices was shown to improve the performance of the dissolved-oxygen sensor. By employing organic precursors, which impart surface hydrophobicity, the quenching response can be comparable to that obtained in the gas phase. The PEG-DA matrices have sufficient density to prevent movement of the two fibers with respect to each other, while still allowing fast oxygen diffusion to the encapsulated ruthenium sensor for fast response times. Thus, the results indicate the potential for remote dissolved-oxygen sensing for use in a wide range of environments.

\section{Acknowledgments}

This work was supported by a grant from the University of Wisconsin Groundwater Research Program. Additional support through a University of Wisconsin-Milwaukee Dissertation Fellowship and a Dr. and Mrs. George Sosnovsky Award for Graduate Research (MVR) are gratefully acknowledged. The authors would like to thank Paul Henning for assistance with writing of the data acquisition software. 


\section{References}

[1] O. S. Wolfbeis, "Fiber-optic chemical sensors and biosensors," Analytical Chemistry, vol. 80, pp. 4269-4283, 2008.

[2] S. M. Borisov and O. S. Wolfbeis, "Optical biosensors," Chemical Reviews, vol. 108, pp. 423-461, 2008.

[3] O. S. Wolfbeis, "Fiber-optic chemical sensors and biosensors," Analytical Chemistry, vol. 78, pp. 3859-3874, 2006.

[4] B. J. Prince, N. T. Kaltcheva, A. W. Schwabacher, and P. Geissinger, "Fluorescent fiber-optic sensor arrays probed utilizing evanescent fiber-fiber coupling," Applied Spectroscopy, vol. 55, no. 8, pp. 1018-1024, 2001.

[5] L. C. Clark and C. Lyons, "Electrode systems for continuous monitoring in cardiovascular surgery," Annals of the New York Academy of Sciences, vol. 102, pp. 29-45, 1962.

[6] O. S. Wolfbeis, Fiber Optic Chemical Sensors and Biosensors, CRC Press, Boca Raton, Fla, USA, 1991.

[7] P. Hartmann, M. J. P. Leiner, and M. E. Lippitsch, "Luminescence quenching behavior of an oxygen sensor based on a $\mathrm{Ru}(\mathrm{II})$ complex dissolved in polystyrene," Analytical Chemistry, vol. 67, no. 1, pp. 88-93, 1995.

[8] G. Orellana, D. García-Fresnadillo, M. D. Marazuela, M. C. Moreno-Bondi, J. Delgado, and J. M. Sicilia, In Monitoring of Water Quality: The Contribution of Advanced Technologies, Elsevier, Amsterdam, The Netherlands, 1998.

[9] H. Xu, J. W. Aylott, R. Kopelman, T. J. Miller, and M. A. Philbert, "A real-time ratiometric method for the determination of molecular oxygen inside living cells using sol-gelbased spherical optical nanosensors with applications to rat C6 glioma," Analytical Chemistry, vol. 73, no. 17, pp. 4124-4133, 2001.

[10] J. N. Demas, B. A. DeGraff, and P. B. Coleman, "Oxygen sensors based on luminescence quenching," Analytical Chemistry, vol. 71, no. 23, pp. 793A-800A, 1999.

[11] F. R. W. Trettnak, In Monitoring of Water Quality: The Contribution of Advanced Technologies, Elsevier, Amsterdam, The Netherlands, 1998.

[12] http://www.oceanoptics.com/products/sensors.asp, http://www.presens.de/.

[13] Y. E. L. Koo, Y. Cao, R. Kopelman, S. M. Koo, M. Brasuel, and M. A. Philbert, "Real-time measurements of dissolved oxygen inside live cells by organically modified silicate fluorescent nanosensors," Analytical Chemistry, vol. 76, no. 9, pp. 24982505, 2004.

[14] B. D. MacCraith, G. O'Keeffe, C. McDonagh, and A. K. McEvoy, "LED-based fibre optic oxygen sensor using sol-gel coating," Electronics Letters, vol. 30, no. 11, pp. 888-889, 1994.

[15] B. D. MacCraith, C. M. McDonagh, G. O’Keeffe et al., "Fibre optic oxygen sensor based on fluorescence quenching of evanescent-wave excited ruthenium complexes in sol-gel derived porous coatings," Analyst, vol. 4, no. 118, pp. 385-388, 1993.

[16] Z. Rosenzweig and R. Kopelman, "Development of a submicrometer optical fiber oxygen sensor," Analytical Chemistry, vol. 67, no. 15, pp. 2650-2654, 1995.

[17] J. R. Lakowicz, Principles of Fluorescence Spectroscopy, Springer, New York, NY, USA, 3rd edition, 2006.

[18] S. L. R. Barker, H. A. Clark, S. F. Swallen, R. Kopelman, A. W. Tsang, and J. A. Swanson, "Ratiometric and fluorescencelifetime-based biosensors incorporating cytochrome c' and the detection of extra- and intracellular macrophage nitric oxide," Analytical Chemistry, vol. 71, no. 9, pp. 1767-1772, 1999.

[19] I. Klimant, F. Ruckruh, G. Liebsch, A. Stangelmayer, and O. S. Wolfbeis, "Fast response oxygen micro-optodes based on novel soluble ormosil glasses," Mikrochimica Acta, vol. 131, no. 1-2, pp. 35-46, 1999.

[20] S. Dourado and R. Kopelman, "Development of fluorescent fiber optic single-polymer membrane sensors for simultaneous ratiometric detection of oxygen and carbon dioxide in biological systems," in Proceedings of the Chemical, Biochemical, and Environmental Fiber Sensors X, R. A. Lieberman, Ed., vol. 3540 of Proceedings of SPIE, pp. 224-234, February 1999.

[21] Y. Kostov, K. A. van Houten, P. Harms, R. S. Pilato, and G. Rao, "Unique oxygen analyzer combining a dual emission probe and a low-cost solid-state ratiometric fluorometer," Applied Spectroscopy, vol. 54, no. 6, pp. 864-868, 2000.

[22] W. Wang, C. E. Reimers, S. C. Wainright, M. Shahriari, and M. J. Morris, "Applying fiber-optic sensors for monitoring dissolved oxygen," Sea Technology, vol. 40, no. 3, p. 5, 1999.

[23] C. S. Chu and Y. L. Lo, "High-performance fiber-optic oxygen sensors based on fluorinated xerogels doped with $\mathrm{Pt}(\mathrm{II})$ complexes," Sensors and Actuators B, vol. 124, no. 2, pp. 376382, 2007.

[24] T. S. Yeh, C. S. Chu, and Y. L. Lo, "Highly sensitive optical fiber oxygen sensor using Pt(II) complex embedded in sol-gel matrices," Sensors and Actuators B, vol. 119, no. 2, pp. 701-707, 2006.

[25] N. Pérez-Ortíz, F. Navarro-Villoslada, G. Orellana, and F. Moreno-Jiménez, "Determination of the oxygen permeability (Dk) of contact lenses with a fiber-optic luminescent sensor system," Sensors and Actuators B, vol. 126, no. 2, pp. 394-399, 2007.

[26] S. Arain, G. T. John, C. Krause, J. Gerlach, O. S. Wolfbeis, and I. Klimant, "Characterization of microtiterplates with integrated optical sensors for oxygen and $\mathrm{pH}$, and their applications to enzyme activity screening, respirometry, and toxicological assays," Sensors and Actuators B, vol. 113, no. 2, pp. 639-648, 2006.

[27] L. Guo, Q. Ni, J. Li et al., "A novel sensor based on the porous plastic probe for determination of dissolved oxygen in seawater," Talanta, vol. 74, no. 4, pp. 1032-1037, 2008.

[28] A. S. Kocincova, S. M. Borisov, C. Krause, and O. S. Wolfbeis, "Fiber-optic microsensors for simultaneous sensing of oxygen and $\mathrm{pH}$, and of oxygen and temperature," Analytical Chemistry, vol. 79, no. 22, pp. 8486-8493, 2007.

[29] N. J. Harrick, Internal Reflection Spectroscopy, Interscience Publishers, New York, NY, USA, 1967.

[30] Y. Ueno and M. Shimizu, "An optical-fiber cable fault location method," IEEE Journal of Quantum Electronics, vol. 11, pp. 899-900, 1975.

[31] Y. Ueno and M. Shimizu, "Optical fiber fault location method," Applied Optics, vol. 15, no. 6, pp. 1385-1388, 1976.

[32] M. K. Barnoski and S. M. Jensen, "Fiber waveguides: a novel technique for investigating attenuation characteristics," Applied Optics, vol. 15, no. 9, pp. 2112-2115, 1976.

[33] S. D. Personick, "Photon prove, an optical-fiber time-domain reflectometer," Bell System Technical Journal, vol. 56, no. 3, pp. 355-366, 1977.

[34] R. A. Potyrailo and G. M. Hieftje, "Optical time-of-flight chemical detection: absorption-modulated fluorescence for spatially resolved analyte mapping in a bidirectional distributed fiber-optic sensor," Analytical Chemistry, vol. 70, no. 16, pp. 3407-3412, 1998.

[35] C. A. Browne, D. H. Tarrant, M. S. Olteanu, J. W. Mullens, and E. L. Chronister, "Intrinsic Sol-Gel Clad fiber-optic sensors with time-resolved detection," Analytical Chemistry, vol. 68, no. 14, pp. 2289-2295, 1996. 
[36] B. J. Prince, A. W. Schwabacher, and P. Geissinger, "A readout scheme providing high spatial resolution for distributed fluorescent sensors on optical fibers," Analytical Chemistry, vol. 73, no. 5, pp. 1007-1015, 2001.

[37] P. Geissinger and A. W. Schwabacher, "Applications of distributed optical fiber sensing: fluorescent assays of linear combinatorial arrays," in Annual Review of Fluorescence, C. D. Geddes and J. R. Lakowicz, Eds., vol. 1, pp. 164-194, Kluwer Academic/Plenum Publishers, New York, NY, USA, 2004.

[38] P. E. Henning, A. Benko, A. W. Schwabacher, P. Geissinger, and R. J. Olsson, "Apparatus and methods for optical timeof-flight discrimination in combinatorial library analysis," Review of Scientific Instruments, vol. 76, no. 6, Article ID 062220, 2005.

[39] J. R. Bacon, "Determination of oxygen concentrations by luminescence quenching of a polymer-immobilized transition-metal complex," Analytical Chemistry, vol. 59, no. 23, pp. 2780-2785, 1987.

[40] D. B. Papkovsky, "New oxygen sensors and their application to biosensing," Sensors and Actuators B, vol. 29, no. 1-3, pp. 213-218, 1995.

[41] B. Valeur, Molecular Fluorescence: Principles and Applications, Wiley-VCH, Weinheim, Germany, 2002.

[42] J. R. Lakowicz and G. Weber, "Quenching of fluorescence by oxygen: a probe for structural fluctuations in macromolecules," Biochemistry, vol. 12, no. 21, pp. 4161-4170, 1973.

[43] R. Banga, J. Yarwood, A. M. Morgan, B. Evans, and J. Kells, "FTIR and AFM studies of the kinetics and self-assembly of alkyltrichlorosilanes and (perfluoroalkyl)trichlorosilanes onto glass and silicon," Langmuir, vol. 11, no. 11, pp. 4393-4399, 1995.

[44] U. Abraham, "Self-assembled monolayers of alkyltrichlorosilanes: building blocks for future organic materials," Advanced Materials, vol. 2, no. 12, pp. 573-582, 1990.

[45] P. Silberzan, L. Léger, D. Ausserré, and J. J. Benattar, "Silanation of silica surfaces: a new method of constructing pure or mixed monolayers," Langmuir, vol. 7, no. 8, pp. 1647-1651, 1991.

[46] D. J. Beebe, J. S. Moore, J. M. Bauer et al., "Functional hydrogel structures for autonomous flow control inside microfluidic channels," Nature, vol. 404, no. 6778, pp. 588-590, 2000.

[47] J. Z. Hilt, A. K. Gupta, R. Bashir, and N. A. Peppas, "Ultrasensitive biomens sensors based on microcantilevers patterned with environmentally responsive hydrogels," Biomedical Microdevices, vol. 5, no. 3, pp. 177-184, 2003.

[48] J. Hoffmann, M. Plötner, D. Kuckling, and W. J. Fischer, "Photopatterning of thermally sensitive hydrogels useful for microactuators," Sensors and Actuators A, vol. 77, no. 2, pp. 139-144, 1999.

[49] A. Revzin, R. J. Russell, V. K. Yadavalli et al., "Fabrication of poly(ethylene glycol) hydrogel microstructures using photolithography," Langmuir, vol. 17, no. 18, pp. 5440-5447, 2001.

[50] M. Lei, Y. Gu, A. Baldi, R. A. Siegel, and B. Ziaie, "Highresolution technique for fabricating environmentally sensitive hydrogel microstructures," Langmuir, vol. 20, no. 21, pp. 8947-8951, 2004.

[51] R. M. Rounds, B. L. Ibey, H. T. Beier, M. V. Pishko, and G. L. Coté, "Microporated PEG spheres for fluorescent analyte detection," Journal of Fluorescence, vol. 17, no. 1, pp. 57-63, 2007.

[52] C. McDonagh, B. D. MacCraith, and A. K. McEvoy, "Tailoring of Sol-Gel films for optical sensing of oxygen in gas and aqueous phase," Analytical Chemistry, vol. 70, no. 1, pp. 4550, 1998.
[53] X. Chen, Z. Zhong, Z. Li, Y. Jiang, X. Wang, and K. Wong, "Characterization of ormosil film for dissolved oxygen-sensing," Sensors and Actuators B, vol. 87, no. 2, pp. 233-238, 2002.

[54] C. McDonagh, C. Kolle, A. K. McEvoy et al., "Phase fluorometric dissolved oxygen sensor," Sensors and Actuators B, vol. 74, no. 1-3, pp. 124-130, 2001.

[55] P.E. Henning and P. Geissinger, "Application of timecorrelated single photon counting and stroboscopic detection methods with an evanescent-wave fibre-optic sensor for fluorescence lifetime-based $\mathrm{pH}$ measurements," Measurement Science and Technology, vol. 23, Article ID 045104, 11 pages, 2012.

[56] D. R. Lide, Handbook of Chemistry and Physics, CRC Press, Boca Raton, Fla, USA, 82nd edition, 2001.

[57] M. V. Rigo, R. J. Olsson, and P. Geissinger, "Crossed-fiber sensors for oxygen measurement and intensity referencing for use in high spatial resolution optical fiber sensor arrays," Sensors \& Transducers, vol. 113, pp. 18-32, 2010.

[58] Z. J. Fuller, W. D. Bare, K. A. Kneas, W. Y. Xu, J. N. Demas, and B. A. DeGraff, "Photostability of luminescent ruthenium(II) complexes in polymers and in solution," Analytical Chemistry, vol. 75, no. 11, pp. 2670-2677, 2003.

[59] P. E. Henning, M. V. Rigo, and P. Geissinger, "Fabrication of a porous fiber cladding material using microsphere templating for improved response time with fiber optic sensor arrays," The Scientific World Journal, vol. 2012, Article ID 876106, 7 pages, 2012.

[60] Z. H. Morton, B. Fabrizio, M. Luca, and L. H. Gordon, "Rate constants for the quenching of excited states of metal complexes in fluid solution," Journal of Physical and Chemical Reference Data, vol. 18, pp. 219-543, 1989.

[61] J. M. Harris, "Introduction to biotechnical and biomedical applications of poly(ethylene glycol)," in Poly(Ethylene Glycol) Chemistry: Biotechnical and Biomedical Applications, J. M. Harris, Ed., pp. 1-14, Plenum Press, New York, NY, USA, 1992.

[62] C. M. V. P. B. van Wachem, T. Beugeling, J. Feijen, A. Bantjes, J. P. Detmers, and W. G. van Aken, "Influence of protein adsorption on interactions of cultured human endothelial cells with polymers," Journal of Biomedical Materials Research, vol. 21, pp. 701-718, 1987.

[63] R. J. Russell, A. C. Axel, K. L. Shields, and M. V. Pishko, "Mass transfer in rapidly photopolymerized poly(ethylene glycol) hydrogels used for chemical sensing," Polymer, vol. 42, no. 11, pp. 4893-4901, 2001.

[64] P. D. Drumheller and J. A. Hubbell, "Densely crosslinked polymer networks of poly(ethylene glycol) in trimethylolpropane triacrylate for cell-adhesion-resistant surfaces," Journal of Biomedical Materials Research, vol. 29, no. 2, pp. 207-215, 1995.

[65] S. I. Jeon and J. D. Andrade, "Protein—surface interactions in the presence of polyethylene oxide. II. Effect of protein size," Journal of Colloid And Interface Science, vol. 142, no. 1, pp. 159-166, 1991. 

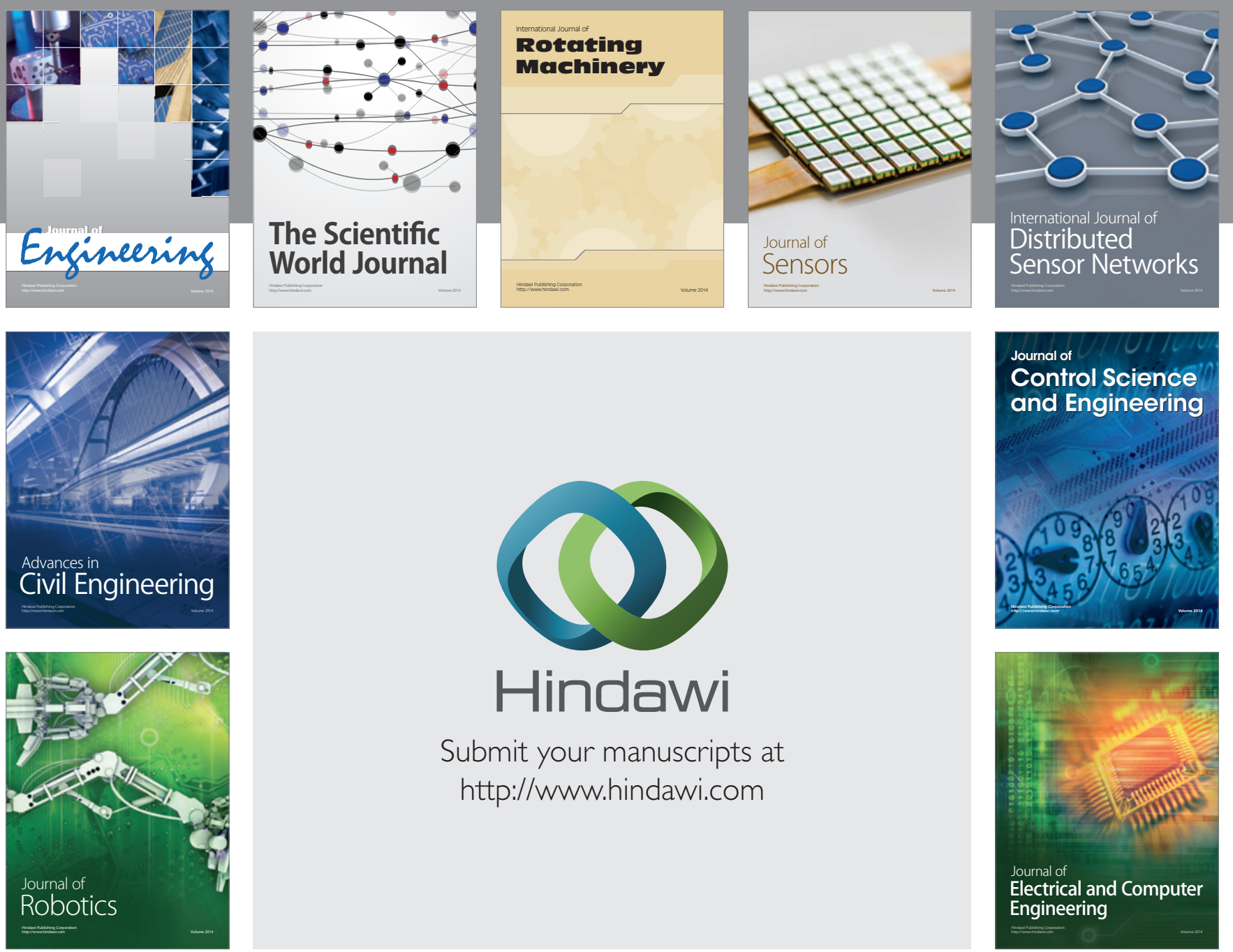

Submit your manuscripts at

http://www.hindawi.com
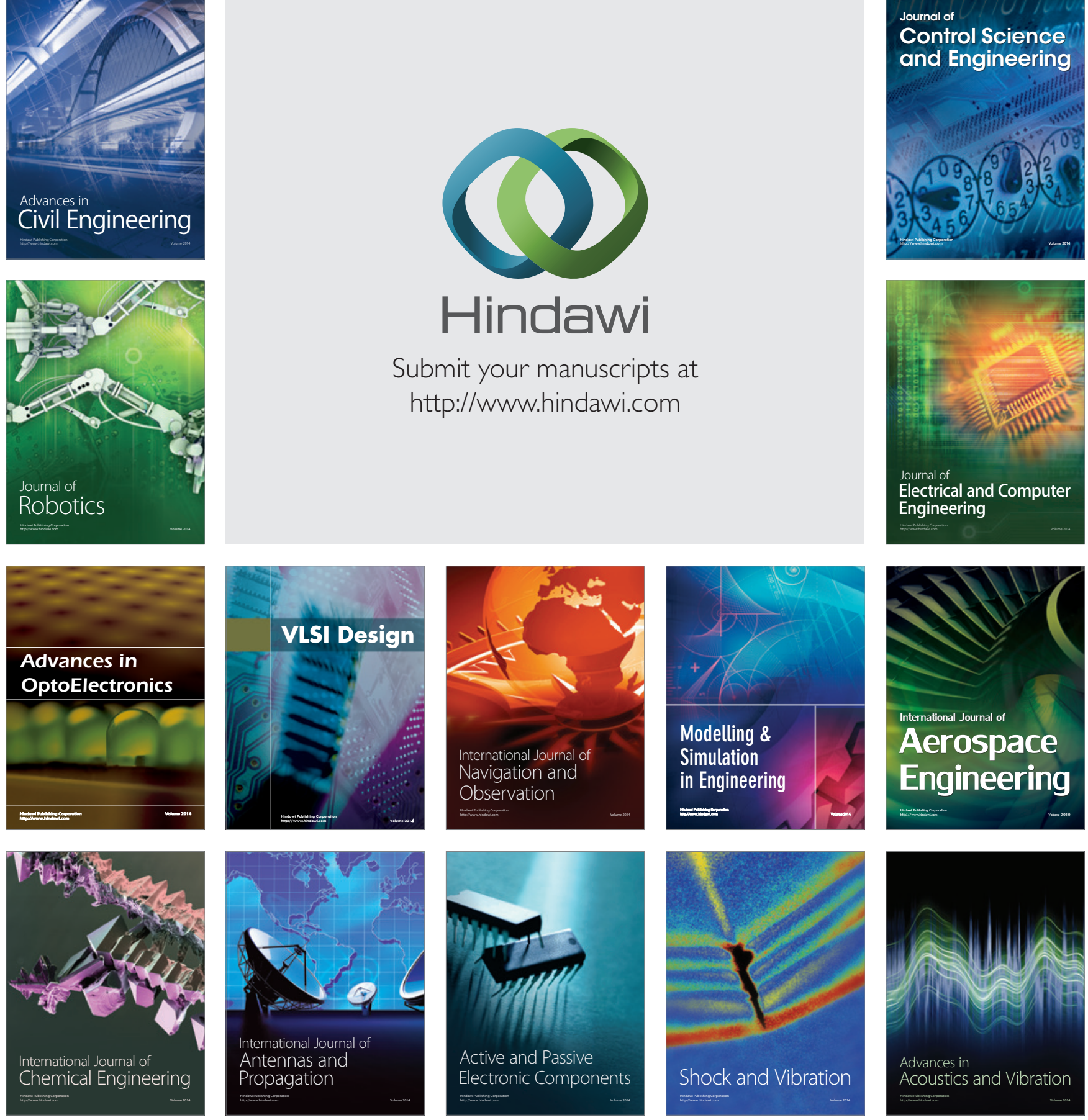